\title{
Between Morphic and Hopfian
}

\begin{abstract}
Relatively morphic submodules are defined and a new class of modules between morphic and Hopfian modules is singled out. Special care is given to the Abelian groups case.
\end{abstract}

\section{Grigore Călugăreanu and Lavinia Pop}

\section{Introduction}

In the 70's, in two successive papers, Gertrude Ehrlich, defined and studied a special class of (von Neumann) regular rings, called unit-regular rings. A ring (with identity) $R$ is unit-regular if for every $a \in R$ there is a unit $u \in U(R)$ such that $a=a u a$. Among other properties, Ehrlich (see [3]) proved the following characterization:

Theorem. Let $A$ be a ring with identity and let $M$ be a right $A$-module such that $R=\operatorname{End}_{A} M$ is a regular ring and let $\alpha \in R$. The following statements are equivalent:

(1) $\alpha$ is unit regular,

(2) there is an automorphism $u: M \longrightarrow M$ such that $\operatorname{Im} \alpha \oplus \mathrm{u} \operatorname{ker} \alpha=\mathrm{M}$,

(3) $\operatorname{ker} \alpha \cong \operatorname{Coker} \alpha$ (here $\operatorname{Co} \operatorname{ker} \alpha=M / \operatorname{Im} \alpha$ ).

This was the starting point for studies concerning the dual of E. Noether isomorphism theorem.

In a series of papers written by K. Nicholson and E. Sanchez-Campos, (see [8], [9]) beginning in 2003, the interest for the dual of E. Noether isomorphism theorem flourished again. In 2004 (see [10]) morphic modules were defined

Key Words: Relatively morphic submodule; $\delta$-module;Abelian $\delta$-groups;Hopfian module. 2010 Mathematics Subject Classification: Primary 16D80, 20K27; Secondary 16D70, 20K10, 20K15.

Received: January, 2012.

Revised: February, 2012.

Accepted: November, 2012. 
via the $R$-endomorphism ring: an endomorphism $\alpha$ was called morphic if $M / \operatorname{im}(\alpha) \cong \operatorname{ker} \alpha$, a module ${ }_{R} M$ was called morphic if every endomorphism is morphic and a ring $R$ was called left (right) morphic provided that ${ }_{R} R$ (or $R_{R}$ respectively) is a morphic module. Among the basic characterizations, we mention the following lattice type (see [10]): a module $M$ is morphic if and only if whenever $M / K \cong H$ where $K$ and $H$ are submodules of $M$, then $M / H \cong K$.

Nowadays, the subject continues to be investigated in several papers, including the determination of morphic Abelian groups, the extension of morphic definition to arbitrary groups, and to categories.

Since morphic Abelian groups turn out to be rare, and Hopfian Abelian groups abound (and so a characterization is out of reach), finding classes of Abelian groups in between seems to be of interest. This is what this paper does.

For the sake of generality, the classes of interest and their general properties are first introduced for modules (called $\delta$-modules and $\gamma$-modules respectively).

A detailed study is made for $\mathbf{Z}$-modules, that is, for Abelian groups.

\section{Relatively morphic submodules}

A submodule $H$ will be called relatively morphic in a module $M$ if whenever $M / K \cong H$ holds for a submodule $K$ of $M$, then $M / H \cong K$.

While 0 is relatively morphic in any module $M$, it is readily checked that

Lemma 1. $M$ is relatively morphic in $M$ if and only if $M$ has no proper factor groups isomorphic with $M$ if and only if it is Hopfian.

Obviously, all submodules in a morphic module are relatively morphic.

Examples. 1) Proper submodules $H<M, H \cong M$ are not relatively morphic. For instance, $n \mathbf{Z}$ is not relatively morphic in $\mathbf{Z}$, for any $n \neq 0,1$.

2) Suppose in a module $M$, there are no proper submodules $H, K$ with $M / K \cong H$. Then (trivially) all proper submodules are relatively morphic in $M$. For instance, since every proper factor group of the full rational group $\mathbf{Q}$ is torsion, all proper subgroups of $\mathbf{Q}$ are relatively morphic in $\mathbf{Q}$ (actually this follows also because $\mathbf{Q}$ is morphic). Moreover, let $A$ be a rank 1 torsion-free Abelian group, i.e., $\mathbf{Z}<A<\mathbf{Q}$. The same argument shows that all proper subgroups in $A$ are relatively morphic in $A$.

3) In $G=\mathbf{Z} \oplus \mathbf{Z}$ there are subgroups (Z-submodules) which are not relatively morphic. Indeed, take $K=\mathbf{Z}$ and $H=n \mathbf{Z}$. Then $(\mathbf{Z} \oplus \mathbf{Z}) / \mathbf{Z} \cong \mathbf{Z} \cong n \mathbf{Z}$ but $G / n \mathbf{Z}=(\mathbf{Z} \oplus \mathbf{Z}) /(0 \oplus n \mathbf{Z}) \cong \mathbf{Z} \oplus \mathbf{Z}(n) \not \mathbf{Z}$. Hence $H=n \mathbf{Z}$ is not relatively morphic in $G$, and so, not all the subgroups of a free Abelian group of finite rank are relatively morphic. 
4) Relatively morphic is not a transitive relation i.e., if $L$ is relatively morphic in $H$ and $H$ is relatively morphic in $M$, then $L$ is not necessarily relatively morphic in $M$.

For a counterexample we can use Example 14 in [10]: there exists a nonmorphic module $M$ with submodule lattice $0 \subset Q \subset P \subset M$. For our purpose, notice that $Q$ is relatively morphic in $P$ and $P$ is relatively morphic in $M$. Indeed, first notice that isomorphic modules have isomorphic submodule lattices. So $P / K \cong Q$ is possible only for $K=Q$ (and this is true via $\left[\begin{array}{l}c \\ d \\ 0\end{array}\right] \longmapsto\left[\begin{array}{l}d \\ 0 \\ 0\end{array}\right]$ ) and so $Q$ is relatively morphic in $P$. Finally, $M / K \cong P$ is not possible for any $K \in\{0, Q, P, M\}$.

5) The conditions [ $H$ is relatively morphic in $M]$ and $[M / H$ is morphic] are logically independent. Indeed,

(i) since $\mathbf{Q}$ is morphic, every subgroup is relatively morphic. So $\mathbf{Z}$ is relatively morphic in $\mathbf{Q}$. However $\mathbf{Q} / \mathbf{Z} \cong \bigoplus \mathbf{Z}\left(p^{\infty}\right)$ is not morphic.

(ii) $\mathbf{Z} / n \mathbf{Z}$ is morphic but $n \mathbf{Z}$ is not relatively morphic in $\mathbf{Z}$ (for $n \neq 0,1$ ).

6) Not all direct summands are relatively morphic.

Example 2. Take $G=\mathbf{Z}(4) \oplus \mathbf{Z}(2)=\langle\{a, b\} \mid 4 a=2 b=0\rangle=A \oplus B$ with $A=\langle a\rangle, B=\langle b\rangle$.

Clearly $G /(2 A \oplus B) \cong B$ but $G / B \cong A \nsubseteq 2 A \oplus B$ (cyclic-Klein). Hence $B$ is (a direct summand) not relatively morphic.

An equivalent definition of relatively morphic submodules using endomorphisms will be useful

Proposition 3. $H$ is relatively morphic in $M$ if and only if every endomorphism $\alpha \in \operatorname{End}(M)$ with im $\alpha=H$ is morphic.

Proof. Indeed, for any endomorphism $\alpha$ (with or without im $\alpha=H$ ), Noether isomorphism theorem holds and so, if $H=\mathrm{im} \alpha$ is relatively morphic, $M / \operatorname{ker} \alpha \cong \operatorname{im} \alpha$ implies $M / \operatorname{im} \alpha \cong \operatorname{ker} \alpha$. Conversely, let $K$ be a submodule of $M$ such that, $M / K \cong H$ and let $\sigma: M / K \longrightarrow H$ be such an isomorphism. Consider the composition $\alpha=i_{H} \circ \sigma \circ p_{K}$ with inclusion $i_{H}: H \hookrightarrow M$ and projection $p_{K}: M \rightarrow M / K$. Then im $\alpha=H$ and $\operatorname{ker} \alpha=K$ and by hypothesis, $M / \operatorname{im} \alpha \cong \operatorname{ker} \alpha$. Hence $M / H \cong K$, as desired.

\section{Property $(\delta)$}

Since $(H / S) \oplus C \cong(H \oplus C) / S$ holds for submodules $H, C$ and $S$ in a module $M$ with $S \leq H$ and $C \cap H=0$, it is readily seen that 
Proposition 4. For modules the following conditions are equivalent:

(i) all the direct summands are relatively morphic, and

(ii) for any direct summand $C$ and submodule $K, M / K \cong M / C$ implies $K \cong C$.

We denote by $(\delta)$ this property. To simplify the wording, such modules are also called $\delta$-modules.

Since an indecomposable module has $(\delta)$ if and only if it is Hopfian, $\mathbf{Z}$ and Q (moreover, all rank 1 torsion-free Abelian groups) satisfy $(\delta)$.

Clearly, morphic modules are $\delta$-modules and $\delta$-modules are Hopfian. Thus we can characterize these three classes by their sets of relatively morphic submodules

$$
\{\text { all submodules }\} \supset\{\text { direct summands }\} \supset\{G\} \text {. }
$$

More important is the following

Proposition 5. $(\delta)$ is inherited by direct summands.

Proof. Suppose $M=H \oplus C$ and $H$ has not property $(\delta)$. Then there are $H=$ $S \oplus T, U \leq H$ with $H / S \cong H / U$ but $S \nsubseteq U$. Clearly $S$ is a direct summand also in $M$ and $U$ is also submodule in $M$. We show that $M / S \cong M / U$ using the first line of this Section. $S, U$ are submodules in $H$ and $H \cap C=0$. Therefore $(H / S) \oplus C \cong(H \oplus C) / S=M / S$ and $(H / U) \oplus C \cong(H \oplus C) / U=M / U$ and so $H / S \cong H / U$ indeed implies, $M / S \cong M / U$.

Since property $(\delta)$ is inherited by direct summands, this property is worth for a study. This is a new class of modules since $\mathbf{Z}$ and Example 2, $\mathbf{Z}(4) \oplus \mathbf{Z}(2)$ (finitely generated and so Noetherian) show that both implications in

$$
\text { morphic } \Longrightarrow(\delta) \Longrightarrow \text { Hopfian }
$$

are not reversible.

Combining Propositions 3 and 4 we obtain

Corollary 6. A module $M$ has $(\delta)$ if and only if every image-direct endomorphism of $M$ is morphic.

It is possible to characterize also Hopfian and Dedekind finite modules respectively, by asking some special endomorphisms to be morphic.

Proposition 7. The following conditions are equivalent

(i) $M$ is Hopfian

(ii) $\operatorname{im} \alpha=M \Longrightarrow \operatorname{ker} \alpha=0$

(iii) every epic endomorphism of $M$ is morphic. 
Proof. Let $\alpha \in \operatorname{End}(M)$ with im $\alpha=M$. If $M$ is Hopfian then $\operatorname{ker} \alpha=0$ and the dual of Noether isomorphism theorem holds: $M / M \cong 0$. Conversely, if the dual of Noether isomorphism theorem holds for an epic endomorphism, $M / M \cong \operatorname{ker} \alpha$ and so $\operatorname{ker} \alpha=0$.

Proposition 8. A module $M$ has a Dedekind finite endomorphism ring if and only if all retractions (right invertible endomorphisms) in $\operatorname{End}(M)$ are morphic.

Proof. Let $\alpha$ be a retraction in $\operatorname{End}(M)$ and $\alpha \beta=1$. Since $\operatorname{End}(M)$ is Dedekind finite, we also have $\beta \alpha=1$ and so $\alpha$ is monic. Hence $M / \operatorname{im} \alpha=$ $M / M \cong 0=\operatorname{ker} \alpha$. Conversely, suppose $\alpha \beta=1$ in $\operatorname{End}(M)$. Since $\alpha$ is a retraction, it is morphic by hypothesis. Therefore ker $\alpha=0$ (as above), $\alpha$ is monic and so an isomorphism ( $\mathbf{A b}$ is a balanced category). Hence $\beta=\alpha^{-1}$ and so $\beta \alpha=1$.

Therefore these four classes of modules can be characterized via their classes of morphic endomorphisms:

$$
\{\text { all endos }\} \supset\{\text { im-direct endos }\} \supset\{\text { epic endos }\} \supset\{\text { retractions }\} \text {. }
$$

\section{Abelian $\delta$-groups}

\subsection{Divisible $\delta$-groups}

Since for $\mathbf{Z}\left(p^{\infty}\right)$ property $(\delta)$ fails, we can prove

Proposition 9. Torsion divisible groups are not $(\delta)$ nor Hopfian.

Proof. Since torsion divisible groups are direct sums of quasicyclic groups and these are indecomposable, these are $(\delta)$ if and only if they are Hopfian. However, $\mathbf{Z}\left(p^{\infty}\right)$ is isomorphic with every proper factor group, so it is not Hopfian, nor $(\delta)$. Therefore, there are no torsion divisible groups with $(\delta)$ nor Hopfian (because both classes are closed to direct summands).

Therefore in all these three classes: morphic, $\delta$, and Hopfian Abelian groups, divisible groups are torsion-free, and so finite direct sums of $\mathbf{Q}$.

However, [torsion-free $\Longrightarrow$ divisible], valid for morphic groups, fails for $(\delta)$ or Hopfian groups: indeed, $\mathbf{Z}$ is $(\delta)$ and (so) Hopfian, but not divisible. Hence we proceed by characterizing some torsion-free $\delta$-groups. 


\subsection{Torsion-free $\delta$-groups}

Since $\mathbf{Z}$ has property $(\delta)$, the first step is

Proposition 10. Finite rank free groups are $\delta$-groups.

Proof. Let $H, K$ be arbitrary subgroups in a finite rank free group $G$ and let $G / H \cong G / K$. Since $r(G)=r(H)+r(G / H)$ and similar for $K$, and all these ranks are finite, $r(G / H)=r(G / K)$ implies $r(H)=r(K)$. But subgroups of free groups are also free and having the same rank, they are isomorphic. Hence $H \cong K$ and the claim follows from Proposition 4 .

Remarks. 1) The condition that at least one of the subgroups $H$ and $K$ should be a direct summand was not used. Thus, finite rank free groups share a stronger property than $(\delta)$ : for every two subgroups $H, K$ in $G, H \cong K$ whenever $G / H \cong G / K$.

2) Being finitely generated, these groups are (also) Hopfian.

3) An infinite rank free group is not Hopfian and so nor $(\delta)$.

All finite rank torsion-free groups are Hopfian (this is due to the rank formula for torsion-free groups). However, since nonisomorphic subgroups may have the same rank, this fails for $\delta$-groups.

In an attempt to characterize completely decomposable (i.e., direct sums of rank 1 torsion-free) $\delta$-groups (of finite rank), we first need the following

Proposition 11. If $\operatorname{Hom}(M, N)=0=\operatorname{Hom}(N, M)$ holds for $\delta$-modules $M$ and $N$, then $M \oplus N$ satisfies ( $\delta$ ).

Proof. According to Corollary 6, a group $G$ has $(\delta)$ if and only if every imagedirect endomorphism $\alpha$ of $G$ is morphic. Using the characterization of morphic endomorphisms given in Lemma 1, [10], and the pattern proof of Lemma 25, [10], the only thing which remains to be verified is that in the hypothesis of the Lemma, if $\lambda \in \operatorname{end}(M \oplus N)=\left[\begin{array}{cc}\operatorname{End}(M) & 0 \\ 0 & \operatorname{End}(N)\end{array}\right]$ is image-direct, and $\lambda=\left[\begin{array}{cc}\alpha & 0 \\ 0 & \beta\end{array}\right]$ in matrix form, then $\alpha$ is image-direct in $M$ and $\beta$ is image-direct in $N$. Since in this case $\operatorname{im} \lambda=\operatorname{im} \alpha \oplus \operatorname{im} \beta$, if $\lambda \in \operatorname{end}(M \oplus N)$ is image-direct, with $M \oplus N=\operatorname{im} \lambda \oplus P=\operatorname{im} \alpha \oplus \operatorname{im} \beta \oplus P_{M} \oplus P_{N}$, clearly both $\operatorname{im} \alpha, \operatorname{im} \beta$ are also image-direct in $M$ and $N$, respectively.

Indeed, then $\alpha^{\prime} \in$ end $M$ and $\beta^{\prime} \in$ end $N$ do exist with $\operatorname{im} \alpha=\operatorname{ker} \alpha^{\prime}$, $\operatorname{ker} \alpha=\operatorname{im} \alpha^{\prime}, \operatorname{im} \beta=\operatorname{ker} \beta^{\prime}$, and $\operatorname{ker} \beta=\operatorname{im} \beta^{\prime}$ and so $\operatorname{im} \lambda=\operatorname{ker} \lambda^{\prime}$ and $\operatorname{ker} \lambda=\operatorname{im} \lambda^{\prime}$, where $\lambda^{\prime}=\left[\begin{array}{cc}\alpha^{\prime} & 0 \\ 0 & \beta^{\prime}\end{array}\right]$. 
Corollary 12. Every finite rank completely decomposable (torsion-free) Abelian group with incomparable type summands is a $\delta$-group.

Example. If $\mathbf{Z}^{(p)}=\left\{\frac{m}{n} \mid(n ; p)=1\right\}$, for any finite set of prime numbers $P, \bigoplus_{p \in P} \mathbf{Z}^{(p)}$ is a completely decomposable $\delta$-group.

In another direction, notice that $G \oplus G$ is a $\delta$-group for $G \in\{\mathbf{Z}, \mathbf{Q}\}$.

Finally, since there plenty of direct summands in direct products which are difficult to handle we leave open the following

Question. Is $\prod_{\alpha \in J} M_{\alpha}$ a $\delta$-group iff each $M_{\alpha}$ is a $\delta$-group ?

We just mention that this question has an affirmative answer for Hopfian modules.

\subsection{Torsion $\delta$-groups}

Since there are no torsion divisible $\delta$-groups, the torsion $\delta$-groups are reduced.

First an expected reduction

Proposition 13. A torsion group has property $(\delta)$ if and only if all its primary components have property $(\delta)$.

Proof. The primary components being direct summands, the conditions are necessary $((\delta)$ is inherited by direct summands).

Conversely, let $G=\bigoplus_{p} G_{p}$ be the decomposition of $G$ into $p$-components. Each subgroup $N$ of $G$ has a corresponding decomposition into primary components $N=\bigoplus_{p} N_{p}$ with $N_{p}$ subgroup of $G_{p}$, for all $p$. Therefore $G / N \cong$ $\bigoplus_{p}\left(G_{p} / N_{p}\right)$.

Now suppose $G / N \cong H$ for a direct summand $H$ of $G$. Using a similar decomposition of $H=\bigoplus_{p} H_{p}, \bigoplus_{p}\left(G_{p} / N_{p}\right) \cong \bigoplus_{p} H_{p}$ and so $G_{p} / N_{p} \cong H_{p}$. Since every $G_{p}$ is $(\delta), G_{p} / H_{p} \cong N_{p}$ and accordingly $G / H \cong N$, as desired.

Therefore, only the $p$-groups with property $(\delta)$ must be singled out.

First we mention the following generalization of Example 2

Lemma 14. If $m<n$ are positive integers, the direct sum $A=\mathbf{Z}\left(p^{m}\right) \oplus \mathbf{Z}\left(p^{n}\right)$ is not $(\delta)$.

Proof. Consider $N=\mathbf{Z}\left(p^{m}\right) \oplus p^{m} \mathbf{Z}\left(p^{n}\right)$ and $H=\mathbf{Z}\left(p^{m}\right) \oplus 0$. Then $A / N \cong$ $\mathbf{Z}\left(p^{m}\right) \cong H$ but $A / H \cong \mathbf{Z}\left(p^{n}\right) \nsucceq N$.

Recall that a direct sum of cyclic $p$-groups is called homogeneous if all its components are isomorphic. 
Theorem 15. A (reduced) p-group $G$ has property $(\delta)$ if and only if it is finite and homogeneous.

Proof. Since the condition is sufficient according to Theorem 26, [10] (such $p$ groups are morphic), suppose a $p$-group $G$ is $(\delta)$ and let $B$ be a basic subgroup of $G$.

We first show that $B$ must be finite and homogeneous, that is, all its Ulm-Kaplansky invariants are zero, with (at most) one exception, say $f_{G}(n)$, which is finite. Indeed, if two different invariants are not zero, say $f_{G}(m) \neq$ $0 \neq f_{G}(n)$ with $m<n$ then the basic subgroup contains a direct summand $\mathbf{Z}\left(p^{m}\right) \oplus \mathbf{Z}\left(p^{n}\right)$, which is pure and bounded in $G$, so a direct summand of $G$. But this contradicts the previous Lemma.

Further on, if the only nonzero invariant is infinite, arguing as above, $G$ contains a direct summand which consists in a direct sum of infinitely many isomorphic cyclic $p$-groups, which is not morphic (not even Hopfian), so again a contradiction.

Finally, if $G$ contains a finite homogeneous basic subgroup, again as pure and bounded, this must be a direct summand of $G$, say $G \cong B \oplus(G / B)$ where $G / B$ is divisible. Since $G / B$ is $(\delta)$ as a direct summand of $G$, we must have $G / B=0$ (there are no divisible $p$-groups with $(\delta)$ ) and so $G=B$ is finite and homogeneous.

Therefore

Corollary 16. A p-group is ( $\delta$ ) if and only if it is morphic. So torsion $\delta$ groups are exactly the torsion morphic groups.

Hopfian.

Example. $\bigoplus_{p \in \mathbf{P}} \mathbf{Z}(p)$ satisfies $(\delta)$. Indeed, $\bigoplus_{p \in \mathbf{P}} \mathbf{Z}(p)$ is morphic, so $(\delta)$ and

Remark. Since Noetherian modules are Hopfian, we could ask whether Noetherian modules satisfy property $(\delta)$. Example 2 shows that this is not the case: $\mathbf{Z}(4) \oplus \mathbf{Z}(2)$ is finitely generated (and so Noetherian and Hopfian) but not a $\delta$-group.

\subsection{Mixed $\delta$-groups}

Just by browsing the known properties for morphic and Hopfian groups (see [2] and [1], [12]) respectively, we can formulate (and answer) some questions:

Proposition 17. If $G$ is $(\delta)$ then so is its torsion part $T(G)$.

Proof. As already noticed $T(G)$ is always reduced. If $T(G)$ is not $(\delta)$ then at least one primary component is not $(\delta)$. According to Theorem 15, a primary 
component is not finite or not homogeneous. As in the proof of the Theorem, one can show that $G$ is not $(\delta)$.

In any splitting mixed $\delta$-group, $G=T(G) \oplus F$, the torsion part and $F \cong$ $G / T(G)$, as direct summands, are also $\delta$-groups. In the reversed direction, recall that in [2], we started with a special case of Lemma 24 from [10], namely

Lemma 18. If the direct sum of $R$-modules $N \oplus K$ is morphic and there exists a R-linear epimorphism $\lambda: K \longrightarrow N$ then $K \cong N \oplus \operatorname{ker} \lambda$.

Revision of the proof of Lemma 24 ([10]), shows that in the above statement, "morphic" can be replaced by $(\delta)$, and so yields the following: if there exists a $R$-linear epimorphism $\lambda: K \longrightarrow N$ and $K$ has no direct summands isomorphic to $N$ then $N \oplus K$ is not $(\delta)$.

Therefore the examples given in [2], may be used in order to show that:

(i) "if both the divisible part $D(G)$ and reduced $R$ are $(\delta)$ then $G=$ $D(G) \oplus R$ is $(\delta)$ ", fails, and

(ii) "if both $T(G)$ and $G / T(G)$ are $(\delta)$ then $G$ is $(\delta)$ ", fails too.

\subsection{Special relatively morphic subgroups}

We may wonder when are the $p$-components in a torsion group and the torsion part in a group, respectively relatively morphic. Here are some results.

Proposition 19. A p-component $P$ is relatively morphic in a torsion group $G$ with only finitely many components if $P$ is Hopfian. The converse holds for any primary component in any torsion group $G$ which satisfies $(\delta)$.

First recall that the subgroup lattice of a direct product of coprime groups is lattice isomorphic with the direct product of the subgroup lattices: if $G_{i}$ $(i \in I)$ are coprime groups, then $L\left(\prod_{i \in I} G_{i}\right) \cong \prod_{i \in I} L\left(G_{i}\right)$. Now we are able to complete the

Proof. To simplify the writing, suppose $P$ is a Hopfian $p$-group and $Q$ is a $q$ group, for different primes $p \neq q$. Since $P$ and $Q$ are coprime, a subgroup $K$ in the direct product (or sum) $P \times Q$ is of the form $K=P_{1} \times Q_{1}$ and $(P \times Q) / K=$ $(P \times Q) /\left(P_{1} \times Q_{1}\right) \cong\left(P / P_{1}\right) \times\left(Q / Q_{1}\right)$. If these are also isomorphic to $P$, a $p$-group, and $Q / Q_{1}$ is a $q$-group, we must have $Q_{1}=Q$ and $P / P_{1} \cong P$. Finally, since $P$ is Hopfian, this gives $P_{1}=0$ and so $(P \times Q) / P \cong Q \cong K$, as desired.

Conversely, every primary component $P$ is a direct summand of (a torsion group) $G$, so it is relatively morphic by $(\delta)$. 
Since it is a direct summand, the torsion part $T(G)$ is relatively morphic in every splitting mixed $\delta$-group $G$.

Comments. (a) In a torsion-free group $G$, a subgroup $H$ is pure if and only if $G / H$ is torsion-free. Thus, for two subgroups $H, K$, if $G / K \cong H$ then $K$ is a pure subgroup in $G$. Hence

Proposition 20. Only pure subgroups can be relatively morphic in a torsionfree group.

From another point of view, if a subgroup $H$ is not pure in $G$ and we can find a (pure) subgroup $K$ with $G / K \cong H$ then $H$ is not relatively morphic (because $G / H$ is not torsion-free, and so $¥ K$ ). Even only for torsion-free groups, finding the subgroups which are isomorphic to suitable quotient groups requires a separate study.

(b) The pure subgroups in a divisible group are divisible and hence direct summands. In finite rank torsion-free divisible groups (these are morphic) all subgroups are relatively morphic. So are the divisible subgroups and, as a special case, so is $D(G)$ the divisible part of a $\delta$-group. In general we can have

Proposition 21. The divisible part $D(G)$ is relatively morphic in an arbitrary group $G$ only if $D(G)$ has finite rank.

Proof. Notice that $K$ must be reduced whenever $G / D(G) \cong K$. To simplify the writing suppose $D(G)=\bigoplus_{\aleph_{0}} \mathbf{Q}$ and for a nonzero reduced group $A$, let $G=A \oplus\left(\bigoplus_{\aleph_{0}} \mathbf{Q}\right)$. Then for $K=A \oplus \mathbf{Q}$ we have $G / K \cong D(G)$ but $G / D(G) \cong$ $A \not K$.

Conversely, we mention

Example 22. $\mathbf{Q}^{n}$ is relatively morphic in $\mathbf{Z} \oplus \mathbf{Q}^{n}$ for every positive integer $n$.

Let $K$ be a subgroup of $G=\mathbf{Z} \oplus \mathbf{Q}^{n}$ such that $G / K \cong \mathbf{Q}^{n}$. Since in this case $G / K$ is, together with $G$, torsion-free, $K$ must be a pure subgroup. More, it must be a direct summand because $G$ is completely decomposable, and its reduced part is homogeneous (see [6]). Therefore, to check the claim we are reduced to $\mathbf{Z} \oplus \mathbf{Q}^{n} \cong K \oplus \mathbf{Q}^{n}$ implies $\mathbf{Z} \cong K$, that is cancellation with $\mathbf{Q}^{n}$. But cancellation by a torsion-free group of finite rank is permitted if the complements are of rank 1 (see Exercise 13, 90, [5]).

Since $D(G)=\mathbf{Q}^{n}$, the divisible part, this can be slightly generalized by

Corollary 23. The finite rank divisible part $D(G)$ is relatively morphic in a torsion-free group $G$ if its reduced part is of rank 1. 
(c) A subgroup $E$ is essential in a group $G$ if and only if $S(G) \leq E$ and $G / E$ is torsion. As special case, if $G$ is torsion-free, then $E$ is essential in $G$ exactly when $G / E$ is torsion. Therefore, for any proper subgroup $K, G / E \not K$. Hence, in a torsion-free group $G$, if for an essential subgroup $E$ there is a subgroup $K$ such that $G / K \cong E$, then $E$ is not relatively morphic in $G$ (the study mentioned above would be necessary also in this case).

\section{$5 \quad$ Simple morphic}

A natural problem can be stated: find the modules $M$ such that for each proper submodule $H$ there is a submodule $K$ such that $M / K \cong H$ but $M / H \neq K$, i.e., the modules with no (proper) relatively morphic submodules (we call such modules, simple-morphic). Obviously, simple modules are also simplemorphic.

Since every idempotent (endomorphism) is morphic, every idempotent not 0 or 1 produces (the image) a proper relatively morphic direct summand. Therefore, the endomorphism $\operatorname{ring} \operatorname{End}(M)$ must have only 0,1 idempotents. Hence $M$ must be indecomposable and we are reduced to the following question: which indecomposable modules are simple-morphic?

Since for Abelian groups indecomposable groups are cocyclic or torsionfree, we mention the following

Proposition 24. $\mathbf{Z}\left(p^{n}\right)$ is not simple-morphic and $\mathbf{Z}\left(p^{\infty}\right)$ is simple-morphic.

Proof. As for the cocyclic, every $\mathbf{Z}\left(p^{n}\right)$ is morphic, and so it is not simplemorphic (unless $n=1$ ). Further, for a proper subgroup $H$, and $K \neq \mathbf{Z}\left(p^{\infty}\right)$, the isomorphism $\mathbf{Z}\left(p^{\infty}\right) / K \cong H$ cannot happen (all proper subgroups are finite, all proper quotients are isomorphic to $\left.\mathbf{Z}\left(p^{\infty}\right)\right)$. Therefore, $\mathbf{Z}\left(p^{\infty}\right)$ is trivially simple-morphic.

What remains, are the torsion-free simple-morphic (indecomposable) groups.

Proposition 25. The only rank 1 torsion-free (indecomposable) simplemorphic group is $\mathbf{Z}$.

Proof. For $\mathbf{Z}$ and for any $n \mathbf{Z}$ we take $K=0$ in our previous definition. As for the rest, let $A$ be a rank 1 torsion-free Abelian group, i.e., $\mathbf{Z}<A \leq \mathbf{Q}$. In Section 2, we have noticed that all proper subgroups in $A$ are relatively morphic in $A$ (clearly $A / K$ is a subgroup in $\mathbf{Q} / K$ and so is also torsion). Hence these groups are not simple-morphic. 
The pathologies for (even) indecomposable torsion-free groups of rank 2 or more are well-known from long time. Thus, finding the simple-morphic of this type requires another separate study.

\section{$6 \quad$ The duality}

We can consider also a symmetric definition for dually relatively morphic submodules: if $M / H \cong K$ then $M / K \cong H$, and these two definitions are not equivalent.

We just list dual properties without all (symmetric) proofs.

While $M$ is dually relatively morphic in any module $M$, it is readily checked that

Lemma 26. 0 is dually relatively morphic in $M$ if and only if $M$ has no proper subgroups isomorphic with $M$ if and only if $M$ is co-Hopfian.

All subgroups in a morphic group are relatively morphic and dually relatively morphic.

Remark. Whenever we have $M / K \cong H$ and $M / H \nsubseteq K$, both $H$ is not relatively morphic and $K$ is not dually relatively morphic.

Question. Find the submodules which are both relatively and dually relatively morphic in a module.

Equivalent definition using endomorphisms:

Proposition 27. $H$ is dually relatively morphic if and only if for every endomorphism $\alpha \in \operatorname{End}(M)$ with $\operatorname{ker} \alpha=H$ is morphic.

Proposition 28. For modules the following conditions are equivalent

(i) $M$ has only dually relatively morphic direct summands and

(ii) for any direct summand $C$ and subgroup $K, K \cong C$ implies $M / K \cong$ $M / C$.

Denote by $(\gamma)$ the property above (also called $\gamma$-modules).

Examples. 1) $\mathbf{Z}$ is not $(\gamma): 2 \mathbf{Z} \cong 3 \mathbf{Z}$ but $\mathbf{Z} / 2 \mathbf{Z}=\mathbf{Z}(2) \varsubsetneqq \mathbf{Z}(3)=\mathbf{Z} / 3 \mathbf{Z}$. (Obvious, also not co-Hopfian)

2) The essential subgroups in a torsion-free Abelian group are (trivially) dually relatively morphic.

Indeed, if $G$ is a torsion-free group then $E$ is an essential subgroup if and only if $G / E$ is torsion. If, for an essential subgroup $E$, there is a subgroup $K \leq G$ such that $G / E \cong K$ then necessarily $K=0$ and $G / E=0$ implies $E=G$. Hence $E=G \cong G / 0=G / K$. 
Therefore all the nonzero subgroups of $\mathbf{Z}$ and $\mathbf{Q}$ are dually relatively morphic. Actually this is clear in the second case, because $\mathbf{Q}$ is morphic.

Clearly morphic modules are $\gamma$-modules and $\gamma$-modules are co-Hopfian. Thus we can characterize these three classes by their sets of dually relatively morphic submodules

$$
\{\text { all submodules }\} \supset\{\text { direct summands }\} \supset\{0\} \text {. }
$$

Since an indecomposable group has property $(\gamma)$ if and only if it is co-Hopfian, $\mathbf{Z}\left(p^{\infty}\right)$ satisfies $(\gamma)$.

This is a new class of modules since $\mathbf{Z}\left(p^{\infty}\right)$ and once again Example 2 show that both implications in

$$
\text { morphic } \Longrightarrow(\gamma) \Longrightarrow \text { co-Hopfian }
$$

are not reversible.

Two simple connections

Proposition 29. Let $M=H \oplus C$. If $M$ is a $\delta$-module and $C$ is dually relatively morphic then $H$ is relatively morphic. If $M$ is a $\gamma$-module and $H$ is relatively morphic then $C$ is dually relatively morphic.

Less favorable than the property $(\delta)$, in order to have the closure for direct summands for $(\gamma)$, we need more.

A module $M$ has cancellation for direct summands if $A \cong B$ whenever $C \oplus A \cong C \oplus B$ with direct summand $C$ and submodules $A, B$.

Proposition 30. $(\gamma)$ is inherited by direct summands, in groups with cancellation for direct summands.

Combining Propositions 27 and 28 we obtain

Corollary 31. A module $M$ has property $(\gamma)$ if and only if every kernel-direct endomorphism $\alpha$ of $M$ is morphic.

It is possible to characterize also co-Hopfian and Dedekind finite modules respectively by asking some special endomorphisms to be morphic.

Proposition 32. The following conditions are equivalent

(i) $M$ is co-Hopfian

(ii) $\operatorname{ker} \alpha=0 \Longrightarrow \operatorname{im} \alpha=M$

(iii) the monic endomorphisms of $M$ are morphic. 
Proposition 33. A module $M$ has a Dedekind finite endomorphism ring if and only if all sections (left invertible endomorphisms) in $\operatorname{End}(M)$ are morphic.

Therefore all these four classes of modules can be characterized via the validity of the dual of Noether isomorphism theorem for some classes of endomorphisms:

$$
\{\text { all endos }\} \supset\{\text { ker-direct endos }\} \supset\{\text { monic endos }\} \supset\{\text { sections }\} .
$$

\subsection{Abelian $\gamma$-groups}

Using integer multiplications as $\mathbf{Z}$-endomorphisms, now we can prove

Proposition 34. Torsion-free $\gamma$-groups are divisible.

Therefore

Corollary 35. The only torsion-free $\gamma$-groups are the finite direct sums of $\mathbf{Q}$.

Corollary 36. Every splitting mixed $\gamma$-group $G$, decomposes as $G=T(G) \oplus$ $D(G)$, with $D(G)$, a finite direct sum of $\mathbf{Q}$.

However the converse, that is, "every divisible $\gamma$-group is torsion-free", fails (take $\mathbf{Z}\left(p^{\infty}\right)$ ). It is considerably harder to characterize torsion $\gamma$-groups mainly because we don't always have cancellation. However, we can prove a structure result for torsion (divisible) $\gamma$-groups.

Proposition 37. The torsion divisible $\gamma$-groups are exactly the divisible torsion groups with finite $p$-ranks (that is, for each prime number $p$, direct sums of finitely many copies of quasicyclic groups (i.e., $\mathbf{Z}\left(p^{\infty}\right)$ ).

Proof. The proof is similar to the one of Proposition 13, because for such groups we have (general) cancellation. Indeed, for (quasi-)injective modules, the cancellation property is equivalent to co-Hopfian (or Dedekind finite) property (see [13] or [7]), and so holds for $\gamma$-groups.

Using the same result, since an Abelian group is quasi-injective if and only if it is divisible or torsion with homogeneous $p$-components (see [4]), we deduce

Corollary 38. Torsion groups with homogeneous p-components are $\gamma$-groups if and only if each p-component is a $\gamma$-group. 
Clearly, each $p$-component must have finite rank (otherwise it is not coHopfian nor Dedekind finite). Finally, since homogeneous $p$-groups of finite rank are morphic, we obtain

Corollary 39. Torsion groups with homogeneous p-components of finite rank are $\gamma$-groups.

Similarly to the way we already used $\mathbf{Z}(4) \oplus \mathbf{Z}(2)$, one can prove

Lemma 40. If $m<n$ are positive integers, the direct sum $A=\mathbf{Z}\left(p^{m}\right) \oplus \mathbf{Z}\left(p^{n}\right)$ is $\operatorname{not}(\gamma)$.

However, although this Lemma is (as Lemma 14 was for Theorem 15) crucial for proving the statement "a (reduced) p-group $G$ has property $(\gamma)$ if and only if it is finite and homogeneous", one can see that final argument in the proof of Theorem $15(G / B$ divisible implies $G=B)$ does not work here.

\section{References}

[1] Baer R. Groups without proper isomorphic quotient groups. Bull. A.M.S. 50 (1944), 267- 277.

[2] Călugăreanu G. Morphic Abelian Groups. Journal of Algebra and Applications 9, no. 2 (2010), 185 - 193.

[3] Ehrlich G. Units and one-sided units in regular rings, Trans. A. M. S. 216 (1976), 81 - 90.

[4] Fuchs L. On quasi-injective modules. Ann. Scuola Norm. Sup. Pisa (3) 23 (1969), 541- 546.

[5] Fuchs L. Infinite abelian groups. Vol. II. Pure and Applied Mathematics. Vol. 36-II. New York-London, Academic Press (1973).

[6] Fuchs L., Kertesz A., Szele T. Abelian groups in which every serving subgroup is a direct summand. Publ. Math. Debrecen 3 (1953), 95 - 105

[7] Lam T. Y. A Crash Course on Stable Range, Cancellation, Substitution, and Exchange. J. Algebra Appl. 3 (2004), 301 - 343.

[8] Nicholson W. K., Sanchez Campos E. Rings with the dual of the isomorphism theorem. J of Algebra 271 (2004), 391 - 406. 
[9] Nicholson W. K., Sanchez Campos E. Principal rings with the dual of the isomorphism theorem. Glasgow M. J. 46 (2004), 181-191.

[10] Nicholson W. K., Sanchez Campos E. Morphic Modules. Comm. in Algebra 33 (2005), 2629 - 2647.

[11] Li Y., Nicholson W.K., Zan L. Morphic groups. Journal of Pure and Applied Algebra, 214, Issue 10 (2010), 1827-1834.

[12] Varadarajan K. Hopfian and co-Hopfian objects. Publ. Mat. 36 (1) (1992), $293-317$.

[13] Warfield R. B. Cancellation of modules and groups and stable range of endomorphism rings. Pacific J. Math. 91, (2) (1980), 457 - 485.

Grigore CĂLUGĂREANU,

Department of Mathematics,

Faculty of Mathematics and Computer Science,

Babeş-Bolyai University,

str. Kogalniceanu 1, 40080 Cluj-Napoca, Romania.

Email: calu@math.ubbcluj.ro

Lavinia POP,

Department of Mathematics,

Faculty of Mathematics and Computer Science,

Babeş-Bolyai University,

str. Kogalniceanu 1, 40080 Cluj-Napoca, Romania.

Email: lavinpop@yahoo.com 